\title{
Are jets in GRS 1758-258 precessing?
}

\section{Pedro Luis Luque-Escamilla*}

Dept. Ingeniería Mecánica y Minera. EPS Jaén. Universidad de Jaén.

E-mail: petereujaen.es

\section{Josep Martí}

Dept. Física. EPS Jaén. Universidad de Jaén.

E-mail: jmarti@ujaen.es

The large-scale morphology of the radio jets in microquasar GRS 1758-258 has been changing in the last decades. Available radio maps show hints of apparent precession. Here, we fit data with a simple kinematical model and perform an analysis of the possible origins and implications of precession in this system. From our study, that includes an additional observing epoch, we are able to confirm the previous detection of changes in the radio jets of GRS 1758-258 far from the core. However, we tentatively suggest that these changes are more probably due to instabilities instead of jet precession.

High Energy Phenomena in Relativistic Outflows VII

9-12 July 2019

Barcelona. SPAIN

\footnotetext{
* Speaker.

$\dagger$ The National Radio Astronomy Observatory is a facility of the National Science Foundation operated under cooperative agreement by Associated Universities, Inc. This work was supported by the Agencia Estatal de Investigación grant AYA2016-76012-C3-3-P from the Spanish Ministerio de Economía y Competitividad (MINECO), by the Consejería de Economía, Innovación, Ciencia y Empleo of Junta de Andalucía under research group FQM-322, as well as FEDER funds.
} 


\section{Introduction}

In the early nineties the GRANAT/SIGMA satellite discovered one of the strongest hard X-ray sources in Universe [1]. It was placed close to the direction of the center of the Galaxy. Named as GRS $1758-258$, its relevance made it worthy to be extensively studied in all wavelengths. The $\mathrm{X}$-ray variability and spectrum suggested it to be a binary with a $\sim 10 \mathrm{M}_{\odot}$ black hole as a compact companion [2, 3]. An orbital period of $18.45 \pm 0.10$ days have been proposed based on Rossi $X$ ray Timing Explorer (RXTE) lightcurve [4], which pointed to a KIV type donor companion. Soon, GRS 1758-258 was considered as a prototypical microquasar when a pair of relativistic, bipolar jets were detected at radio frequencies, with two remarkable lobes spanning a projected distance of 2.5 arcmin [5, 6]. However, the optical/infrared counterpart remained elusive for decades because of the high extinction, the crowded field and some astrometric uncertainties [7, 8, 9, 10, 11]. Finally, a clear candidate was proposed [12,13,14], which seems to be compatible with an A5V star orbiting the black hole in less than a day, clearly in contradiction with the commonly accepted companion spectral type and orbital period. Unfortunately, this counterpart is so difficult to observe that no clear spectrum has been obtained up to now. Therefore, much of the true nature of the GRS 1758258 system is still unknown.

Nevertheless, changes in the large-scale morphology of the GRS 1758-258 radio jets have been noticed recently [13]. This definitively proved the Galactic origin of the system, and posed a new quasar/microquasar analogy in the cocoon features due to the interaction between the jets and the interestellar medium. The Z-shaped observed morphology in such cocoon for GRS 1758258 was intriguing because it seemed to mimic winged radiogalaxies. This new link between microquasars and their extragalactic relatives has been proposed and reported in detail no long ago [15], with some consequences in the black hole merger rate for extragalactic sources and their corresponding gravitational wave production.

\section{Precessing model for GRS 1758-258 relativistic jets}

The morphological changes observed in the large-scale radio jets of GRS 1758-258 were initially attributed to the growth of Kelvin-Helmholtz (KH) and/or Rayleigh-Taylor (RT) instabilities [13]. However, the evolution seen on the shape of the radio outflow along time seem to follow helical paths typical of precessing jets, such as those in SS 433. In order to explore this possibility, we decided to add a new radio map to the existing ones in the databases and ask for observation time to the Very Large Array (VLA) in C- configuration. A new observing run was finally conducted in 2016 at the $6 \mathrm{~cm}$ wavelength (Project ID. 16A-005, on-source time $7659 \mathrm{~s}$ ), with an increased sensitivity with respect to the historical data thanks to the $\mathrm{GHz}$ bandwidth available after the last VLA upgrade. Calibration and imaging were performed taking care of producing a comparable map with the preceding ones, following the methodology we usually adopt (see [16]). The final complete set of radio maps is presented in Fig. 1 together with the result of a simultaneous fit using the well-known kinematic model of Hjellming \& Johnston [17] with the same set of parameters for all the maps. This is shown in Table 1. As may be seen, the modeled jet paths seem to be compatible with the observed radio emission. Therefore, the possibility for the jet morphological evolution to be due to precession must be considered and discussed. 


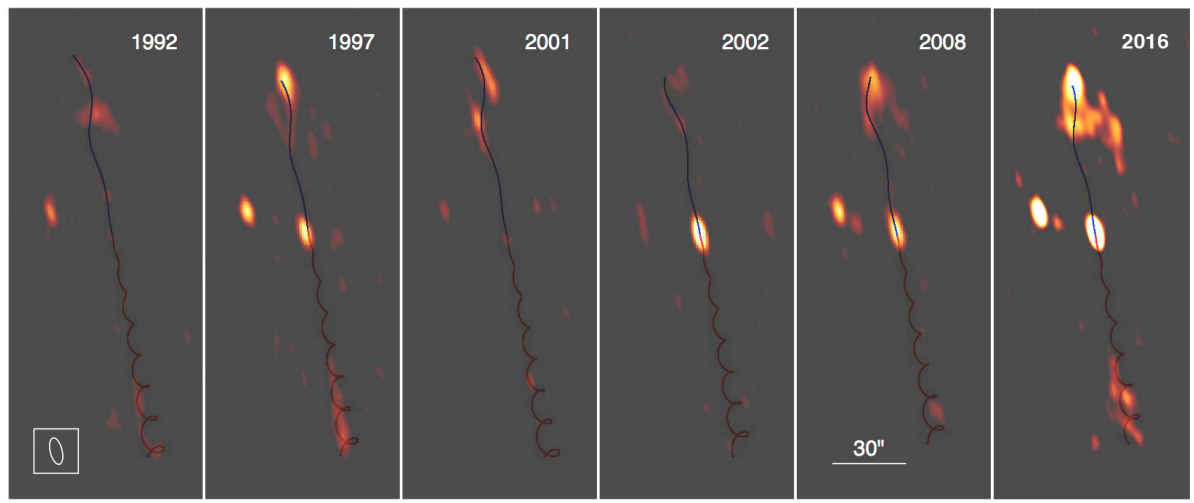

Figure 1: Attempt to fit the large-scale radio jets of GRS 1758-258 jets at different epochs using a theoretical kinematic model [17]. Horizontal bar shows the scale size, and the synthesized beam is on the left-down corner. North is up and East is left.

Table 1: Twin-jet kinematic model for GRS 1758-258 radio jets(*)

\begin{tabular}{ll}
\hline \hline Parameter & Value \\
\hline Angle of the precession cone & $\psi=1.4^{\circ}$ \\
Inclination of the jet precession & $i=34^{\circ}$ \\
axis with the 1.o.s. & \\
Inclination of jet normal axis to North & $\chi=281^{\circ}$ \\
& (posit. angle $=11^{\circ}$ ) \\
Approaching jet (N) & $s_{\text {jet }}=+1$ \\
Receding jet (S) & $s_{\text {jet }}=-1$ \\
Sense of rotation (clockwise) & $s_{\text {rot }}=-1$ \\
Precession period & $P_{p}=1099 \mathrm{~d}$ \\
Jet velocity & $v_{\text {jet }}=0.67 c$ \\
Distance & $d=8.5 \mathrm{kpc}$ \\
\hline \hline
\end{tabular}

(*) Adapted from 1981ApJ...246L.141H.

\section{Possible mechanisms for precession: misalignment of black hole rotation axis with respect to orbital angular momentum}

Assuming jets in GRS 1758-258 are launched via the Blandford-Znajek mechanism [18], they will lay along the black hole rotation axis via Bardeen-Petterson effect or magneto-spin alignment [19]. Thus, for precession to take place, a misalignment of this axis with respect to the orbital angular momentum must be persistent along time. However, both axes try to realign in a time scale of [20]:

$$
\tau_{\text {align }}[y r]=1.0 \times 10^{7} a^{11 / 16}\left(\frac{\alpha}{0.03}\right)^{13 / 8}\left(\frac{L}{0.3 L_{E d d}}\right)^{-7 / 8}\left(\frac{M_{\bullet}}{10 M_{\odot}}\right)^{-1 / 16} \times\left(\frac{\varepsilon}{0.3}\right)^{7 / 8}\left(\frac{t_{r}}{t_{o}}\right)
$$

where $a$ is the dimensionless spin parameter for the black hole, $\alpha$ is the viscosity parameter [21], $L$ is the luminosity of the accretion flow, $L_{E d d}$ is the Eddington luminosity for the central black hole, $t_{r}$ and $t_{o}$ are the recurrence and outburst time-scales of the central source, respectively, and $\varepsilon$ is the 
radiative efficiency of the accretion flow. This last parameter is related to $a$ according to [22] :

$$
\varepsilon=1-\frac{1-2 \xi_{m s}^{-1}+a \xi_{m s}^{-3 / 2}}{\sqrt{1-3 \xi_{m s}^{-1}+2 a \xi_{m s}^{-3 / 2}}} .
$$

Here, $\xi_{m s} \equiv R_{m s} / R_{g}$, with $R_{m s}$ being the marginally-stable-orbit radius, and $R_{g}=G M_{\bullet} / c^{2}$ the gravitational radius of the black hole. This is in turn related to $a$ as $\xi_{m s}=3+A_{2} \mp\left[\left(3-A_{1}\right)\left(3+A_{1}+\right.\right.$ $\left.\left.2 A_{2}\right)\right]^{1 / 2}$ (upper sign for prograde rotation), with $A_{1}=1+\left(1-a^{2}\right)^{1 / 3}\left[(1+a)^{1 / 3}+(1-a)^{1 / 3}\right]$ and $A_{2}=\left(3 a^{2}+A_{1}^{2}\right)^{1 / 2}[23]$.

On the other hand, the age of the binary system (identified as the timescale to accrete the entire donor star, which is an upper bound for the lifetime of the Roche lobe overflow of the binary) is of the order of [20]:

$$
\tau_{\text {bin }}[y r]=1.3 \times 10^{8} q\left(\frac{\varepsilon}{0.3}\right)\left(\frac{L}{L_{E d d}}\right)^{-1}\left(\frac{t_{r}}{t_{o}}\right),
$$

where $q$ is the mass ratio $M_{\text {donor }} / M_{\bullet}$. Here, we will assume that GRS 1758-258 has $M_{\text {donor }} \sim 2$ $\mathrm{M}_{\odot}$ (typical of a A5V star) and $M_{\bullet} \sim 10 \mathrm{M}_{\odot}$, while $L \sim 0.03 L_{E d d}$ [3] and $a \sim 0.1$ (typical for a radio-quiet source [24]). This gives $\varepsilon=0.06$, and using a typical $\alpha \sim 0.01$ value, we obtain $\tau_{\text {align }} / \tau_{\text {bin }} \sim 10^{-1}$. Even taking into account that the donor star lifetime $\tau_{*} \sim 2 \times 10^{10}$ years [25] could be one order of magnitude less than $\tau_{\text {bin }}$, the alignment timescale is of the order of the system life. As, in addition, $\tau_{\text {align }}$ is greater than the jet travel time $\tau_{j} \lesssim 10^{5}$ years [15], the black hole spin axis in GRS 1758-258 could be misaligned with the orbital orbital momentum, as usual in X-ray binaries with black holes [20], giving rise to precession.

\section{Possible mechanisms for precession: Bardeen-Petterson}

If jets are launched by Blandford \& Payne [26] mechanism, they will be always perpendicular to the accretion disc plane no matter the spin of the black hole. Thus, misalignment of the jets causing precession may be due to a very different mechanism: the Bardeen-Petterson effect [23]. Here, a local warping of the inner part of the disc forces it to stay perpendicular to the black hole spin axis in its proximity if viscosity is high enough. This may be quantified through the viscous and precession timescales that may be expressed as $\tau_{v}=r^{2} v_{2}(r)^{-1}$ and $\tau_{p}=\pi c^{3} r^{3} a^{-1} G^{-2} M_{\bullet}^{-2}$, respectively, with $v_{2}(r)$ being the kinematic viscosity in transverse direction, and $G$ the gravity constant. If $\tau_{p} / \tau_{v} \lesssim 1$ at the inner edge of the accretion disc the Bardeen-Petterson precession may take place [27]. We can assume the inner radius of the disc at $r \sim R_{m s}$, and that $v_{2}$ is of the order of the radial viscosity, which may be parametrized in terms of the local sound speed [21], which in turn depends on the disc scale heigh $H$. Taking into account the values yet adopted for GRS 1758-258 we can write:

$$
\frac{\tau_{p}}{\tau_{v}}=10^{-1}\left(\frac{\alpha}{0.05}\right)\left(\frac{a}{0.1}\right)^{-1}\left(\frac{r}{R_{m s}}\right)^{3 / 2}\left(\frac{H / r}{0.1}\right)^{2},
$$

where we have assumed a very conservative estimate for $H / r$ at the inner border of the disc, despite which the Bardeen-Petterson precession could be taken place in GRS 1758-258. 


\section{Possible mechanisms for precession: tidal torque}

A third mechanism could explain the precession of jets in GRS 1758-258 if orbital and accretion disc planes were misaligned in a Blandford-Payne launching jet scenario. Torques thus arising may cause the bipolar jets to precess [28, 29, 30, 31, 32]. This mechanism has been proposed for X-ray binaries, and it could be very well at work for the case of GRS 1758-258.

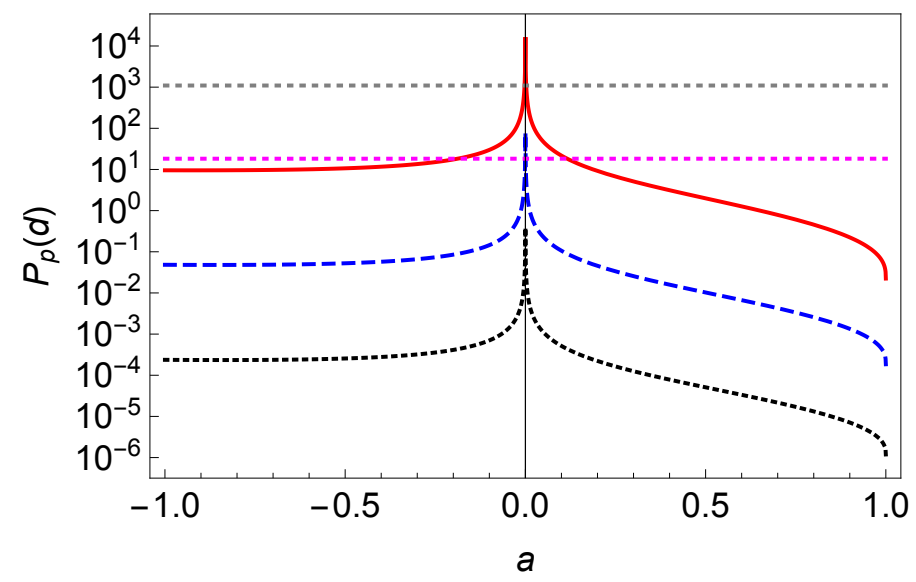

Figure 2: The precession period for GRS $1758-258$ as a function of the spin parameter. The outer nondimensional radius of the disc is taken to be $\xi_{\text {out }}=1000 \xi_{m s}$. Different lines correspond to the different power-law slopes of the disc surface density (red, continuous for $s=0$; blue, dashed for $s=-1$ and black, dotted for $s=-2$ ). The gray, dotted horizontal line marks the here estimated period of precession of approximately $3 \mathrm{yr}$, while the magenta, dotted line marks the 18.48 days precession period proposed in [14], both in the comoving frame of the source.

\section{Proposed scenario: precession and instabilities}

It is clear that precession may be taking place in our system. The three mechanisms here discussed are consistent with such a possibility. However, is precession at GRS 1758-258 actually responsible for its observed radio-jets evolution? If so, the precession period should be of the order of the one obtained in our fit (see Table 1), which is of about 3 years. Therefore, we have to analyze in deep the precession period compatible with any of the previously presented models.

Our first case associated to a Blandford-Znajek jet lunch mechanism is very difficult to reconcile with this period, because the precession must be related to the orbital period, which is far from a yearly timescale. On the other hand a Bardeen-Petterson precession would lead to a precession period [33] of:

$$
P_{p}=\frac{2 \pi G M_{\bullet}}{c^{3}} \frac{\int_{\xi_{\text {out }}}^{\xi_{\text {in }}} \Sigma(\xi)[\Phi(\xi)]^{-1} \xi^{3} d \xi}{\int_{\xi_{\text {in }}}^{\xi_{\text {out }}} \Sigma(\xi) \Psi(\xi)[\Phi(\xi)]^{-2} \xi^{3} d \xi},
$$

where $\Phi(\xi)=\xi^{3 / 2}+a, \Psi(\xi)=1-\left(1-4 a \xi^{-3 / 2}+3 a^{2} \xi^{-2}\right)^{1 / 2}$ and $\Sigma(\xi)$ is the accretion disk surface density, and $\xi$ is a the radius non-dimensionalised with $R_{g}$. Let assume again $\xi_{\text {in }}=\xi_{m s}$ and that $\Sigma(\xi)$ is a potential function with exponent $s \in\{-2,-1,0\}$ to model different decreasing (or constant) behaviors. We will take $\xi_{\text {out }}=10^{3} \xi_{m s}$ as in [14] and numerically integrate the previous 
equation to obtain the precession period as a function of $a$ and $s$ as may be seen in Fig. 2. As a result, we can check that the $\sim 3$ years period of our fitting is only compatible with a too slow spinning black hole. However, it is noteworthy that a more realistic result compatible with previously assumed $a=0.1$ appears when assuming a precession of the order of the canonical orbital period of $\sim 18$ days. It may seems awkward to make such an hypothesis, but some facts supports it. For instance, many low mass X-ray binaries (e.g. Her-X1 [34], SMC X-1 [35]) show a slight variability in their precession periods, and the same seems to be the case of GRS 1758-258 [36], with $P_{p}$ varying from 18.04 to 18.47 days. In addition, the BlackCAT catalogue of stellar black holes in X-ray transients [37] contains data that clearly show that almost all the measured orbital periods for low mass X-ray binaries are of $\sim 1$ day or even shorter.

In any case, both solutions need a constant density disc. It is possible that our hypothesis on the disc size was wrong, but a smaller disc would lead to shorter, less reasonable periods. A larger disc compatible with the Sakura-Sunyaev solution $s=-3 / 4$ for disc density together with the $a=0.1$ black hole spin may be found, but for the $\sim 3$ year precession period it leads to a too unrealistic outer radius of $10^{5} R_{g}$, which is too close to the binary separation and would lead to a brighter emission from the disc. On the other hand, a precession period of 8 days leads to a much more adequate outer radius of $8.5 \times 10^{3} R_{g}$.

Our last considered scenario to support precession in GRS 1758-258 was based on tidal torque. The precession period $P_{p}$ may be related to orbital period $P_{o}$ through $[28,38]$ :

$$
\frac{P_{o}}{P_{p}}=\frac{3}{7} \frac{q}{\sqrt{1+q}}\left(\frac{0.49}{0.6+q^{2 / 3} \ln \left(1+q^{-1 / 3}\right)}\right)^{3 / 2} \kappa^{3 / 2} \cos \delta .
$$

Here, the inclination $\delta$ of the binary orbit with respect to the accretion disk plane gives rise to the observed precession. The jet then subtends a cone with a half-opening angle equal to the orbit inclination, so $\delta=\psi$, which we adopt from the model fit ( $\psi=1.4^{\circ}$; see Table 1$)$. A $\kappa \equiv$ $R_{\text {out }} / R_{\text {Rochelobe }}$ value of $\sim 0.86$ is taken from literature $[39,40]$. If the classical companion KIV spectral type is assumed, with $P_{o}=18.45$ days, then the $P_{p} \sim 3$ years of our fit would lead to $P_{o} / P_{p}=0.017$, which is half of the theoretical value obtained from Equation 6.2. However, the scenario proposed by the authors with an A5V companion and $P_{o} \sim 0.6$ day $[12,13,14]$ together with the assumption of $P_{p}=18.45$ days would lead to a $P_{o} / P_{p}$ very much closer to the one derived from Equation 6.2. In addition, the same theory [28] leads to a too large accretion disc for being compatible with observed emission if the classical KIV scenario is assumed.

Therefore, although precession is probable in GRS 1758-258 system, there is a clear lack of consistency between the theoretically acceptable and the fitted value of the precession period. On the other hand, instabilities seem to be a natural explanation for the evolution of the radio jets in GRS 1758-258, as has been already pointed out [16]. RT and KH instabilities grow with timescales of the same order of magnitude [16, 41], which for GRS 1758-258 is of about 800 days or more. Current driven instabilities growth timescale is $\tau_{C D} \sim 10 R_{\text {jet }} / v_{A}$ [42], with $v_{A}$ being the Alfvén velocity. Assuming equipartition and ultrarelativistic plasma, and a measured jet radius $R_{j}=0.1$ pc, $\tau_{C D} \simeq 1.5 \times 10^{3}$ days. The helical pattern in the 1997 map in Fig. 1 has a wavelength $\lambda \sim 0.62$ $\mathrm{pc}$ at the assumed distance of $8.5 \mathrm{kpc}$ to the source. Thus, the CD kink instability would have a reasonable $v_{j} \sim \lambda / \tau_{C D} \sim 0.5 c$ but only if it should start very close to the onset of the helical structure. And this seems problematic, because $\mathrm{CD}$ instabilities need to be triggered very near the 
black hole, where the magnetic fields are too strong. However, KH instabiliites may start far from the central engine, and the same reasoning would account for $v_{j} \sim \lambda / \tau_{K H} \sim c$ which is reasonable for a lower $\tau_{K H}$ limit. So, instabilities such as these, or even the recently proposed centrifugal one [43] (that may appear in the case of curved jets confined by an external medium at rest) could explain the observed evolution in GRS 1758-258 radio jets.

\section{Conclusions}

We can conclude that the GRS 1758-258 system is probably composed of an A5V companion and a $\sim 10 \mathrm{M}_{\odot}$ black hole with an orbital period $P_{o} \sim 0.6$ days. The radio jets could be precessing with a period $P_{p}=18.45$ days, the up-to-now assumed orbital period. But this short precession period would lead to too wiggled structures in radio maps, which are not observed. Therefore, as previously proposed [16], the growth of instabilities seems to be the cause of the changing morphology along years in the radio maps of GRS 1758-258. All this discussion is being the matter of a paper in a near future.

\section{References}

[1] R. Sunyaev, E. Churazov, M. Gilfanov, M. Pavlinsky, S. Grebenev, G. Babalyan et al., Two hard X-ray sources in 100 square degrees around the Galactic Center, 247 (1991) L29.

[2] D. S. Main, D. M. Smith, W. A. Heindl, J. Swank, M. Leventhal, I. F. Mirabel et al., Long-Term X-Ray Monitoring of IE 1740.7-2942 and GRS 1758-258, 525 (1999) 901 [astro-ph/ 9906178 ].

[3] R. Soria, J. W. Broderick, J. Hao, D. C. Hannikainen, M. Mehdipour, K. Pottschmidt et al., Accretion states of the Galactic microquasar GRS 1758-258, 415 (2011) 410 [1103.3009].

[4] D. M. Smith, W. A. Heindl and J. H. Swank, Orbital and superorbital periods of 1e 1740.7-2942 and grs 1758-258, The Astrophysical Journal Letters 578 (2002) L129.

[5] L. F. Rodríguez, I. F. Mirabel and J. Martí, The radio counterpart of the hard X-ray source GRS 1758-258, 401 (1992) L15.

[6] I. F. Mirabel, L. F. Rodríguez, B. Cordier, J. Paul and F. Lebrun, A double-sided radio jet from the compact Galactic Centre annihilator 1E1740.7-2942, 358 (1992) 215.

[7] G. F. Bignami, P. A. Caraveo, S. Mereghetti, J. Paul, B. Cordier, A. Goldwurm et al., Optical identification of celestial high energy sources, The Messenger 66 (1991) 10.

[8] S. Mereghetti, P. Caraveo, G. F. Bignami and T. Belloni, Optical imaging of the fields of the two hard X-ray sources in the galactic center region - IE 1740.7-2942 and GRS 1758-258, 259 (1992) 205.

[9] J. Marti, S. Mereghetti, S. Chaty, I. F. Mirabel, P. Goldoni and L. F. Rodriguez, Search for the optical and infrared counterpart of GRS 1758-258, 338 (1998) L95 [astro-ph/9809270].

[10] S. S. Eikenberry, W. J. Fischer, E. Egami and S. G. Djorgovski, Deep Infrared Imaging of the Microquasars IE 1740-2942 and GRS 1758-258, 556 (2001) 1 [astro-ph/0103422].

[11] D. M. Rothstein, S. S. Eikenberry, S. Chatterjee, E. Egami, S. G. Djorgovski and W. A. Heindl, The Infrared Counterpart of the Microquasar GRS 1758-258, 580 (2002) L61 [astro-ph/0210350]. 
[12] A. J. Muñoz-Arjonilla, J. Martí, P. L. Luque-Escamilla, J. R. Sánchez-Sutil, E. Sánchez-Ayaso, J. A. Combi et al., Identification of the optical and near-infrared counterpart of GRS 1758-258, 519 (2010) A15 [1006.4954].

[13] P. L. Luque-Escamilla, J. Martí and Á. J. Muñoz-Arjonilla, The Variable Near-infrared Counterpart of the Microquasar GRS 1758-258, 797 (2014) L1.

[14] J. Martí, P. L. Luque-Escamilla and Á. J. Muñoz-Arjonilla, Optical spectroscopy of the microquasar GRS 1758-258: a possible intermediate mass system?, 596 (2016) A46 [1611.03683].

[15] J. Martí, P. L. Luque-Escamilla, V. Bosch-Ramon and J. M. Paredes, A galactic microquasar mimicking winged radio galaxies, Nature Communications 8 (2017) 1757.

[16] J. Martí, P. L. Luque-Escamilla, G. E. Romero, J. R. Sánchez-Sutil and Á. J. Muñoz-Arjonilla, Real-time evolution of a large-scale relativistic jet, 578 (2015) L11 [1505. 07641 ].

[17] R. M. Hjellming and K. J. Johnston, An analysis of the proper motions of SS 433 radio jets, 246 (1981) L141.

[18] R. D. Blandford and R. L. Znajek, Electromagnetic extraction of energy from Kerr black holes, 179 (1977) 433.

[19] J. C. McKinney, A. Tchekhovskoy and R. D. Blandford, Alignment of magnetized accretion disks and relativistic jets with spinning black holes, Science 339 (2013) 49 [https://science.sciencemag.org/content/339/6115/49.full.pdf].

[20] T. J. Maccarone, On the misalignment of jets in microquasars, 336 (2002) 1371 [astro-ph/0209105].

[21] N. I. Shakura and R. A. Sunyaev, Black holes in binary systems. Observational appearance., 24 (1973) 337.

[22] S. L. Shapiro and S. A. Teukolsky, Black holes, white dwarfs, and neutron stars: The physics of compact objects. 1983.

[23] J. M. Bardeen and J. A. Petterson, The Lense-Thirring Effect and Accretion Disks around Kerr Black Holes, 195 (1975) L65.

[24] R. D. Blandford, H. Netzer, L. Woltjer, T. J.-L. Courvoisier and M. Mayor, eds., Active Galactic Nuclei, 1990.

[25] R. Kippenhahn and A. Weigert, Stellar Structure and Evolution. 1990.

[26] R. D. Blandford and D. G. Payne, Hydromagnetic flows from accretion discs and the production of radio jets, 199 (1982) 883.

[27] P. Natarajan and P. J. Armitage, Warped discs and the directional stability of jets in active galactic nuclei, 309 (1999) 961 [astro-ph/9812001].

[28] J. Larwood, On the precession of accretion discs in X-ray binaries, 299 (1998) L32 [astro-ph/9806348].

[29] J. D. Larwood, The tidal disruption of protoplanetary accretion discs, 290 (1997) 490 [astro-ph/9705252].

[30] J. C. B. Papaloizou and C. Terquem, On the dynamics of tilted discs around young stars, 274 (1995) 987.

[31] J. Papaloizou and D. Lin, On the dynamics of warped accretion disks, ApJ 318 (1995) . 
[32] J. I. Katz, Thirty-five-day periodicity in her x-1, Nature Physical Science 246 (1973) 87 EP .

[33] A. Caproni, H. J. Mosquera Cuesta and Z. Abraham, Observational Evidence of Spin-induced Precession in Active Galactic Nuclei, 616 (2004) L99 [astro-ph/ 0410450 ].

[34] R. Staubert, D. Klochkov, D. Vasco, K. Postnov, N. Shakura, J. Wilms et al., Variable pulse profiles of Hercules X-1 repeating with the same irregular 35 d clock as the turn-ons, 550 (2013) A110 [1212.5472].

[35] K. C. Dage, W. I. Clarkson, P. A. Charles, S. G. T. Laycock and I.-C. Shih, Long-term properties of accretion discs in X-ray binaries - III. A search for spin-superorbital correlation in SMC X-1, 482 (2019) 337 [1809.07335].

[36] M. Hirsch, Unevenly spaced time series analysis: Grs 1758-258, Master's thesis, Erlangen Centre for Astroparticle Physics. Friedrich-Alexander-Universitat, Erlangen-Nurenberg, 1, 2014.

[37] J. M. Corral-Santana, J. Casares, T. Muñoz-Darias, F. E. Bauer, I. G. Martínez-Pais and D. M. Russell, BlackCAT: A catalogue of stellar-mass black holes in X-ray transients, 587 (2016) A61 [1510.08869].

[38] P. P. Eggleton, Approximations to the radii of Roche lobes, 268 (1983) 368.

[39] B. Paczyǹski, A model of accretion disks in close binaries, 216 (1977) 822.

[40] J. Papaloizou and J. E. Pringle, Tidal torques on accretion discs in close binary systems, 181 (1977) 441.

[41] A. T. Araudo, V. Bosch-Ramon and G. E. Romero, High-energy emission from jet-clump interactions in microquasars, 503 (2009) 673 [0906.4803].

[42] Y. Mizuno, Y. Lyubarsky, K.-I. Nishikawa and P. E. Hardee, Three-dimensional Relativistic Magnetohydrodynamic Simulations of Current-driven Instability. III. Rotating Relativistic Jets, 757 (2012) 16 [1207.4949].

[43] K. N. Gourgouliatos and S. S. Komissarov, Reconfinement and loss of stability in jets from active galactic nuclei, Nature Astronomy 2 (2018) 167. 